\title{
WAR-TIME STREET LIGHTING AND AIDS TO MOVEMENT IN STREET*
}

\begin{abstract}
Summary of the Report on Fundamental Requirements, underlying the War-time Street Lighting prepared for the Joint Committee of the Ministry of Home Security and the Illuminating Engineering Society. The Report will appear in full in the Society's " Transactions."
\end{abstract}

The human eye can adjust itself to wide variations in degrees of brightness. The illumination received from summer sunlight may attain 10,000 ft.c., faint starlight may be of the order of $1 / 10,000 \mathrm{ft}$.c. - a range of about one hundred millions to one.

This study is devoted to vision at the lowest levels in this range of illumination -which is all that is available out of doors-on a clear moonless night and is of the same order as that furnished by war-time street lighting ( 0.0002 ft.c.).

\section{Behaviour of the Eye in Weak Light}

The conditions characteristic of " twilight" vision are broadly as follows :-

(1) Complete dark-adaptation is only slowly attained by an eye previously adapted to normal illuminations. A person emerging from a brightly lighted room into a dark street requires some minutes before the eye can " take in " its surroundings. Thirty minutes is a reasonable time to allow for full adaptation. Such adaptation can be quickly destroyed. Momentary exposure of the eye to bright light will partly undo the results of a long period of waiting. Persons differ much in their speed of adaptation. Some, e.g., elderly people, approach the condition of " night blindness" and have great difficulty in finding their way in semi-darkness.

(2) At normal illuminations vision is most acute near the centre of the retina, which is normally exercised in the recognition of form. With twilight vision this area is relatively insensitive. Two consequences follow :-

(i) Objects are no longer perceived by direct vision, but " out of the tail of the eye."

(ii) The outlines of objects are never seen with the same clarity as by normal vision; they tend to appear as masses, limits of which are less clearly defined.

(3) At the low order of illumination characteristic of twilight vision, colours can no longer be distinguished.

* Reprinted from " Light and Lighting," December, 1940, with permission of Mr. J. S. Dow, Secretary of the Illuminating Engineer Society. 


\section{Visual Sensitivity at Low Levels of Examination State of Adaptation}

The provision of even a weak degree of general illumination shortens the period of adaptation and fixes the order of brightness to which the eye must adjust itself, having thus a certain stabilising influence. When exposed to a certain illumination-even if only of a low order-the hyper-sensitiveness of the eye characteristic of complete dark adaptation is less marked and the effect of exposure to an object of moderate brightness less severe. Therefore, the first essential is to abstain from any action liable to impair sensitiveness of the retina. Not merely should extreme conditions of dazzle, creating momentary semi-blindness, be avoided; one should also avoid any condition of excessive contrast such as may depress the action of the eye and prevent it getting the full value of the weak illumination available.

\section{Limits TO BRIGHTNESS}

At very low illuminations new standards are necessary. At normal illuminations, say, about $5 \mathrm{ft} . c$., there are recommendations that within the direct range of vision $(a)$ the brightness of no object should exceed 5 candles per square inch, and that $(b)$ the variation in brightness of objects within this range should not exceed $100: 1$.

Application of the rule quoted above to conditions met with in areas receiving an illumination of $0.002 \mathrm{ft}$.c. would give a limiting brightness of 0.002 candles per square inch, roughly equivalent to $0 \cdot 1$ equivalent foot-candles. Adoption of the 100 to 1 ratio, on the other hand, would give a brightness of about one-fifth of this, namely 0.02 e.f.c.

\section{Size of Object}

At these weak illuminations seeing is effected by the peripheral region of the retina, where perception of form is less acute. Only the broad shapes of things at close quarters can be seen. The image of an object subtending at the eye an angle less than 2 to 3 degrees is liable to fall inside-the central region of the retina when it becomes invisible. Objects of this size cannot be seen with certainty. They " appear and disappear" as one is looking at them. Moreover, impressions may depend upon the product of area and brightness. Larger objects appear brighter than small ones, which again enhances their visibility.

\section{IMPORTANCE OF CONTRAST}

Another factor of vital importance is the ratio of brightness between object and its background. At high illuminations, under 
very favourable conditions, a difference in brightness of only one to two per cent. can be detected. At very weak illuminations a difference of 20 or even 30 per cent. can scarcely be detected with certainty. An object of quite substantial size which reflects only 20 per cent. less or more than the adjacent roadway and receives an illumination of only $0.0002 \mathrm{ft}$.c. might easily escape recognition.

In order to ensure that an object may be visible it is therefore expedient (i) to ensure, if possible, that the angle subtended at the eye is well outside 3 degrees; (ii) to secure a relatively high contrast with its background, and at the same time (iii) to guard against the possibility of depressing vision by excessive contrast, so great as to approach glare conditions.

\section{Colour}

It is evident that it is of no use, at a very low order of brightness, to rely on colour as a means of distinction, since colour as such can no longer be distinguished. Moreover, a red object, such as a pillar box, becomes indistinguishable from a dark background. Objects desired to stand out from their dark surroundings at low illuminations, and which are identified by their colour under normal conditions, should preferably be painted green or blue. The sensitiveness of the eye to blue light is accentuated at very low illuminations. Yet the use of such light in preference to white light is not advantageous. The general use of pure blue light involves considerable absorption, and is therefore uneconomical, and for some purposes it is considered inexpedient owing to certain loss in sharpness of outline of objects illuminated when viewed from some little distance away.

\section{Applications}

The utility of an illumination of the order of $0.0002 \mathrm{ft} . c$. depends entirely on the avoidance of factors liable to depress visual sensitivity. It is desirable to do everything possible to facilitate the transition from the brightness indoors to the relative obscurity outside. Light traps, besides their primary object of preventing escape of light from within, have a secondary field of utility-in providing a period of adaptation. If behind an entrance there were a short passage or ante-room where the illumination was graduated by steps from the full illumination inside (say, 3-5 ft.c.) down to, say, $0.02 \mathrm{ft} . c$. at the threshold where, if possible, one should linger for a short time the final transition down to $1 / 10,000$ th or less of the original illumination would be rendered easier and the period of subsequent adaptation shortened. In any case, a wait of a few minutes is desirable before movement is made.

The next step is to avoid the presence of objects of undue brightness out of doors. Headlights and traffic control signals, the power 
of which is necessarily fixed at a value far exceeding that necessary for the recognition of objects at short range, inevitably give rise to some degree of glare. In both cases, however, control of the beam and the adoption of screens and masks does much to mitigate the trouble. It is chiefly when regulations are disregarded that serious glare is liable to arise.

\section{OBjects OF "UNDUe BRightness"}

For all classes of users, however, the presence of unduly bright objects in the field of view is a drawback. Indications of limiting brightness have been quoted. At this low level of illumination higher values might perhaps be permitted. It is, however, interesting to observe that the first of these values $(0 \cdot 1$ e.f.c.) is the maximum assigned to illuminated A.R.P. signs (BS/ARP 32) as a result of long and continued and careful experimental investigation, whilst the second is exactly that specified in the Lighting Restriction Order as the limiting brightness of illuminated display signs. Hence there seems good ground for the adoption of a value of brightness of the order of 0.05 to 0.1 e.f.c. as a limit in streets -especially as these limits have been independently determined with a view to restricting conspicuousness from the air.

It may be useful to apply this yardstick to luminous objects commonly met with in streets. Reference has been made above to motor car headlights and luminous traffic signals. The position in regard to rearlights and side lights is possibly different. Their effectiveness is properly defined in terms of brightness limited by the use of suitable diffusing media. Other devices, such as the use of a concealed light under the body of the car showing its breadth by silhouette or the application of indirect lighting to the back surfaces of cars, might well be explored and the liability to glare diminished.

Lights indicating the positions of refuges and obstructions frequently offend by their glare, making it difficult to distinguish outlines of adjacent objects. The Restrictions on Lighting Order No. 74, 1940, specifies a brightness for the "cross" of 0.5 candles per sq. ft. (roughly 1.5 e.f.c.), which is 15 times the extreme limit proposed above. (Experience suggests that this value is often greatly exceeded.) The same applies to red lights indicating obstructions. The limitation to 1 c.p. (probably also little observed in practice) is not accompanied by any restriction in brightness, and the presence of such lights may actually impede observation of the limits of the obstruction. From a visual standpoint requirements would be better met by ensuring that objects are provided with a low illumination enabling them to be recognisable, and that the brightness of any signal light, intended to give warning at a distance, does not exceed the limits specified above. 
The torches commonly used by pedestrians have likewise great drawbacks. The concentrated beam they provide produces an illumination at once uneven and in excess of that needful. They would be more serviceable if constructed in a box form with no lens but a light-giving area of several square inches giving diffused light of low brightness but even quality, and equipped with a hood in order to render the emission of light above the horizontal improbable.

\section{Application of Theory to Size and Contrast of ObJects in Streets}

At weak illuminations a degree of contrast much beyond that ordinarily sufficient may be necessary in order to enable even objects of substantial size to be seen. The images of objects subtending less than about $3^{\circ}$ at the eye are liable to be received on the insensitive area and escape notice. Now $3^{\circ}$ represents about $2 \frac{1}{2}$ $\mathrm{ft}$. at a distance of $60 \mathrm{ft}$. It is, therefore, expedient that obstructions should be illuminated over an area having at least this linear dimension. It is also desirable to increase the available brightness whenever it is expedient to do so. By selecting materials of high reflecting power we may sometimes achieve results as good as if ten times the illumination on a relatively dark material were provided.

The area of darkest tone commonly met with in the street is the road surface. A city pavement, in general, comes conveniently about mid-way between this and white paint, and therefore affords a moderately good background both for the figures of persons in dark clothing and for obstacles treated with this material. White paint used with discretion at busy crossings and on vertical surfaces such as walls and railings, etc., enables persons crossing the roadway to be more easily seen.

The extensive whitening of the kerb both on the horizontal, and for drivers of vehicles and persons on the other side of the road, the value of the whitening of the vertical surfaces of kerbs has proved itself. The application of white paint to motor vehicles is also useful, but should be applied to a much greater area than is at present usual.

Contrasts in brightness play an important part in creating a sense of perspective and enabling people to "size up" their position. This is illustrated by the admitted value under conditions of war-time street lighting of the circular white rings on tree trunks. There seems every reason to exploit to the full the revealing value of white surfaces placed at corners, and similar devices which show up in a headlight beam. Wider use might also be made of directive reflecting surfaces, as commonly used for warning signs, etc. 
On the other hand, isolated bright spots of light, such as the luminous crosses on islands (and, in some cases, the rear lights of motor vehicles), may, unless carefully controlled, destroy perpective and may render it most difficult for the user of the roadway to see what is beyond them.

\section{Controlled local Brightness}

There have been suggestions in regard to various forms of local lighting (e.g., restricted beams of light.) Experience seems to have proved the fundamental value of a uniform level of illumination with, so far as possible, no regions of complete darkness.

The measure of illumination provided by war-time street lighting, though so small, is of appreciable benefit, especially when applied on a large scale. It affords a ground work on which the eye can build up impressions from any extra casual light it may receive.

It would, however, add to amenities in the streets if somewhat higher illuminations, under proper control, or suitably screened, could be adopted locally in certain instances.

\section{ANNOTATION}

\section{The Empty Socket}

It has often struck us as odd that one does not find an ophthalmological subject used as an Inn sign in this country, but we do not know of a single case. Other anatomical subjects occur: e.g., the hand often, the heart, occasionally. St. Peter's finger is met with in one instance. Arms are, of course, frequently found in this connexion, but here the significance is heraldic and not anatomical. The eye is very rarely met with in heraldry as we have observed before.

Care would be needed in giving an ophthalmic name to a pub, for if the house were called the "evil eye" the more superstitious of the village inhabitants would give it a wide berth. And "the squinting eye" would almost certainly become in local parlance the "boss eye" and from that to "boozy," the transition is easy. Hardy would seem to have anticipated in a mild degree our need, for one of the village inns in "Tess" was called the "Pure Drop." We fear that in this case he was thinking of a drop of drink and not of an eye drop.

The late Mr. George Pollock, in lecturing on fractures at St. George's Hospital more than half a century ago, used to tell an apocryphal story of a certain publican who changed the name of his 